\title{
Time to get our teeth into reducing obesity: should dentists screen and deliver interventions to reduce obesity in the population?
}

\author{
Amanda J. Daley ${ }^{1}$
}

\section{Key points}

As we emerge from the COVID-19 pandemic, it is more important than ever before to reduce obesity in the population.
Through the NHS Making Every Contact Count campaign, all healthcare professionals should aim to improve patients' health behaviours within consultations.
Dental care teams could be vital in screening for obesity and offering weight management interventions.

\begin{abstract}
Little attention has been devoted to how dentists and dental teams may be able to contribute to reducing obesity, by screening for obesity in the population and offering weight management interventions to those who might benefit. Drawing on the NHS Making Every Contact Count campaign, this paper presents a case as to why dentists have an instrumental role in contributing to the global public heath effort to reduce obesity in both adults and children. This paper suggests how dentists might learn lessons from GPs and practice nurses about how to best address and raise the topic of weight management within patient consultations. Lastly, this report offers some tangible plans of action for further research on this question.
\end{abstract}

\section{Introduction}

As we emerge from the COVID-19 pandemic, it is more important than ever before that we test and implement effective population-based interventions to address obesity, particularly in primary healthcare settings. Over the past decade, there has been a focus on testing weight management programmes that are delivered by general practitioners (GPs) and/ or practice nurses on the basis that they have the potential to reach a large proportion of the population, and patients are more likely to make changes to their diet and physical activity when these topics are raised by their doctor or nurse. There have also been campaigns such as NHS Making Every Contact Count in England,

'Professor of Behavioural Medicine, The Centre for Lifestyle Medicine and Behaviour (CLiMB), Loughborough University, Loughborough, UK.

Correspondence to: Amanda Daley

Email address: a.daley@lboro.ac.uk

Refereed Paper.

Accepted 28 July 2021

https://doi.org/10.1038/s41415-022-3872-z which aim to use the millions of everyday interactions that healthcare professionals have with patients, where they can routinely signpost, promote and support improvements in health behaviours, to reduce the risk of disease in the population. ${ }^{1}$ While it does make good public health sense to consider how GPs might usefully screen for obesity and thereafter deliver weight management intervention to the public, this strategy has largely overlooked the important contribution that other key primary care health professionals might have in contributing to reducing population levels of obesity, namely dentists and dental teams.

\section{What is the case for primary care dental teams to screen and deliver weight management interventions?}

The case for dentists to be involved in reducing population obesity is strong. Like GPs, dentists are part of primary care health services and are ideally positioned to screen and intervene to reduce obesity. Dentists typically consult with 22 million adults every two years and 7 million children yearly, meaning they could routinely embed efficient monitoring of weight into dental health services and offer interventions at scale to both adults and children. ${ }^{2}$ Dentists already deliver health behaviour change messages for oral health; for example, smoking cessation, and will counsel patients (particularly children) about reducing or limiting their consumption of sugar and sugar-sweetened beverage intake, which are important contributors to obesity and related diseases. ${ }^{3,4}$ Obesity is associated with diabetes and dental professionals routinely question patients on whether they have been diagnosed with diabetes because of its link with gum disease. ${ }^{5}$ Dental practitioners should therefore have a vested interest in the management of obesity. Dentists have expressed a willingness to intervene to reduce obesity, ${ }^{6,7}$ and studies have reported that the public would be open to receiving support about weight management from dental practitioners. ${ }^{8}$ Pilot trials have reported that weight management interventions for young people delivered by dental teams were well received by both parties. ${ }^{9,10}$ 


\section{Barriers to delivering weight management interventions in dental settings}

The most significant barriers to dental teams offering obesity services are likely to be a perceived lack of time, feeling ill-equipped to deliver weight management interventions, and discomfort about raising the topic of weight because of fear of offending and upsetting patients. ${ }^{11}$ Concern about raising the topic of weight is understandable because conversations about weight are sensitive and difficult. Evidence has also indicted that dentists are reticent about measuring height and weight and often view obesity as a medical issue, rather than a dental concern. ${ }^{7,11}$ Nevertheless, we have seen in the case of GPs that these types of barriers can be resolved relatively easily with continued professional development and practice at having conversations with patients about weight, and we know brief conversations about weight can be effective. ${ }^{12}$ Furthermore, contrary to expectations, patients who are overweight are not usually offended by health professionals raising the topic of weight with them and would welcome support. ${ }^{12}$ An important barrier to the integration of obesity services within routine dental practice will be financial reimbursement, and if this is the case, dentists could be incentivised to screen and/ or intervene, which may also be cost-effective. This said, there has been a lack of high-quality research about how best to integrate effective weight management interventions into dental care settings and this has undoubtedly been a barrier to implementation.

\section{Next steps}

While excess weight is a risk factor for systemic and oral disease, no worldwide public health strategy has been put in place for dentists to screen and/or treat obesity. Changing people's diet and physical activity behaviours is difficult and dental teams have the ideal platform on which to convey healthy weight messages to the public, and the NHS Making Every Contact Count campaign provides a framework for implementing this. If we are serious about reducing obesity in the population, it will require all hands on deck and this will need to involve active advocacy from dental health professionals.

\section{Future research endeavours}

With colleagues, I hope to develop a programme of work over the coming two years to investigate the feasibility of screening for obesity within dental care settings, and thereafter delivering brief weight management interventions to the public in this context. It is also imperative that research assesses the views of both dental teams and patients (children and adults) of doing so. If you are interested in getting involved in this work with us, please get in touch - Twitter: @ Amanadadaley@09 or via our centre e-mail: climb@lboro.ac.uk.

\section{Ethics declaration}

No conflict of interest to declare.

\section{Author contributions}

Amanda Daley is the sole author of this report. Amanda conceived the idea, wrote and approved the paper.
Amanda Daley is supported by a National Institute for Health Research (NIHR) Research Professorship award. The views expressed are those of the author and not necessarily those of the NHS, the NIHR or the Department of Health and Social Care.

\section{References}

1. NHS Health Education England. Making Every Contact Count (MECC). Available at https://www. makingeverycontactcount.co.uk/ (accessed February 2020).

2. NHS Digital. NHS Dental Statistics for England 2018-2019. 2019. Available at https://digital.nhs.uk/ data-and-information/publications/statistical/nhs-dentalstatistics/2018-19-annual-report-pas (accessed July 2021).

3. Rana H, Mallet M C, Gonzalez A, Verreault M F, St-Pierre S. Free Sugars Consumption in Canada. Nutrients 2021; 13: 1471.

4. Miller C, Ettridge K, Wakefield M et al. Consumption of sugar-sweetened beverages, juice, artificially-sweetened soda and bottled water: An Australian population study. Nutrients 2020; 12: 817.

5. NHS. Commissioning standard: dental care for people with diabetes. 2019. Available at https://www.england.nhs.uk/ wp-content/uploads/2019/08/commissioning-standarddental-care-for-people.pdf (accessed May 2021).

6. Curran AE, Caplan D J, Lee J Y et al. Dentists' attitudes about their role in addressing obesity in patients: a national survey. J Am Dent Assoc 2010; 141: 1307-1316.

7. Greenberg B L, Glick M, Frantsve-Hawley J, Kantor M L. Dentists' attitudes toward chairside screening for medical conditions. JAm Dent Assoc 2010; 141: 52-62.

8. Wijey T, Blizard B, Louca C, Leung J, Suvan J. Patient perceptions of healthy weight promotion in dental settings. J Dent 2019; DOI: 10.1016/j.jjodo.2019.100002.

9. Tavares M, Chomitz V. A healthy weight intervention for children in a dental setting: a pilot study. J Am Dent Assoc 2009; 140: 313-316.

10. Watt R G, Crawley H, Pikhart H et al. Preventing obesity in young people attending primary dental care settings: an exploratory randomised controlled trial. 2010. Available at https://fundingawards.nihr.ac.uk/award/PB-PG-120714085 (accessed May 2021).

11. Wright R, Casamassimo PS. Assessing attitudes and actions of paediatric dentists toward childhood obesity and sugar-sweetened beverages. J Public Health Dent 2017; 77 Suppl 1: S79-S87.

12. Aveyard P, Lewis A, Tearne S et al. Screening and brief intervention for obesity in primary care: a parallel, twoarm, randomised trial. Lancet 2016: 388: 2492-2500. 